\title{
Generation of New Fractals via SP Orbit with s-Convexity
}

\author{
Sudesh Kumari $^{\# 1}$, Mandeep Kumari ${ }^{\# 2}$, Renu Chugh ${ }^{\# 3}$ \\ ${ }^{1,2,3}$ Department of Mathematics, \\ Maharshi Dayanand University, \\ Rohtak - 124001, Haryana, India. \\ tanwarsudesh10@gmail.com, \\ ${ }^{2}$ kumarimandeep@gmail.com, \\ 3hugh.r1@gmail.com
}

\begin{abstract}
The generation of fractals using iterative procedures is the emerging and interesting field of research. The aim of this paper is to obtain some fixed point results in the generation of fractals using SP-iterative process with s-convexity for quadratic, cubic and higher degree polynomials. Our results extend and improve the corresponding recent results proved by other authors in the literature.
\end{abstract}

Keywords: Julia set, Mandelbrot set, SP-orbit, s-convexity, escape criterion, complex polynomials.

\section{INTRODUCTION}

Fractal theory is a popular branch of mathematical art. In mathematical visualization they look very beautiful even though they can be created using simple patterns. By zooming a fractal, one can find that the patterns and shapes will continue repeating, forever. In 1919, French mathematician Gaston Julia [9] derived the Julia set when he was studying Cayley's problem related to the behaviour of Newton's method in complex plane. In 1975, Mandelbrot [3] extended the work of Julia and introduced Mandelbrot set. During that time, various Julia and Mandelbrot sets have been introduced for quadratic ([11],[20],[26]) cubic ([1],[2],[4],[5],[14],[17],[20],[23]) and higher degree polynomials [37] using Picard iterative process (a one step feedback process) $[20]$.

In 2004, Rani and Kumar ([18],[19]) introduced the superior iterate and created superior Julia and Mandelbrot sets for quadratic and cubic polynomials. In 2009, D. Rochon [7] studied generalized Mandelbrot sets in bicomplex plane. Later on the work of Rochon was extended by Wang ([33]-[36]) jointly with others and carried further analysis of generalized Julia and Mandelbrot sets. Also, they have studied the fractal structure and discontinuity law of the generalized Julia sets generated from the extended complex mapping $\mathrm{z}^{\mathrm{n}}+\mathrm{c}$, where $\mathrm{n} \in \mathrm{R}[32]$.

This paper deals with $s$-convexity and approximate convexity. Our work is the generalization of the results of Bernstein and Doetsch [8]. The concept of $s$-convexity and rational $s$-convexity was introduced by Breckner and Orban [31]. In 1978, Breckner and Orban [31], Hudzik and Maligranda [10] proved that $s$-convex functions are nonnegative when $0<s<1$; moreover, the set of $s$-convex functions increases as the value of $s$ decreases. In 1994, Hudzik and Maligranda [10] discussed a few results related with $s$-convex functions in the second sense. Some new results about Hadamard's inequality for $s$-convex functions were discussed by Alomari and Darus [12, 13] and Kirmaci et al. [27]. In 1999, Dragomir and Fitzpatrick [25] proved a variant of Hermite Hadamard's inequality for s-convex functions in the second sense. Takahashi [30] first introduced a notion of convex metric space, which is a more general space, and each linear normed space is a special example of this space. In 2010, Rana et al. [21] discussed the dynamics of Ishikawa iteration procedure. Further, Ojha and Mishra [6] discussed an application of a fixed point theorem for s-convex function.

Recently, M. Kumari et al. [16] used a new iterative process (an example of four-step feedback process) for creating new Julia and Mandelbrot sets for quadratic, cubic and higher degree polynomials. Further, some researchers obtained fixed point results in the generation of Julia and Mandelbrot sets with s-convexity, see ([15],[22],[28]). 
In 2011, W. Phuengrattana and S. Suantai [29] proposed the SP-iteration for approximating fixed point of continuous functions on an arbitrary interval. They compared the convergence speed of Mann, Ishikawa, Noor and SP-iterations using some numerical examples and proved that the SP-iteration is equivalent to and converges faster than the other iterations.

In this paper, we obtain some fixed point results in generation of fractals using SP iterative procedure with sconvexity, which is an example of a four-step feedback process. In Section 2, we give some definitions, which are the basis of our work. Section 3 deals with the escape criterions for quadratic, cubic and $n^{\text {th }}$ degree polynomials under the new iteration process. In section 4, we present new Mandelbrot sets and Julia sets with $\mathrm{s}$ - convexity using the software Mathematica 9.0. Finally, section 5 contains the conclusion of our work.

\section{PRELIMINARIES}

Definition 2.1 (Orbit) [20] Given $x_{0} \in R$, then the orbit of $x_{0}$ under the mapping $\mathrm{F}$ is defined to be the sequence of points $x_{0}, x_{1}=F\left(x_{0}\right), x_{2}=F^{2}\left(x_{0}\right), \ldots, x_{n}=F^{n}\left(x_{0}\right), \ldots$

The point $x_{0}$ is called the seed of the orbit.

Definition 2.2 (Julia Set) [9] The Julia set of a function $g$ is the boundary of the set of points $z \in C$ that tend to infinity under repeated iteration by $g(z)$, i.e. the Julia set of the function $g$ is

$$
J(g)=\partial\left\{z \in C: g^{n}(z) \rightarrow \infty \text { as } n \rightarrow \infty\right\}
$$

where $C$ is the complex space, $g^{n}(z)$ is $n^{\text {th }}$ iterate of function $g$.

Definition 2.3 (Mandelbrot Set) [3] The Mandelbrot set $M$ consists of all parameters for which the Julia set of $g$ is connected, that is

$$
M=\{c \in C: J(g) \text { is connected }\} .
$$

In fact, $M$ contains an enormous amount of information about the structure of Julia sets. The Mandelbrot set $M$ for the Quadratic $P_{c}(z)=z^{2}+c$ is defined as the collection of all $c \in C$ for which the orbit of the point 0 is bounded,

$$
\text { i.e., } \quad M=\left\{c \in C:\left\{P_{c}^{n}(0)\right\} ; n=0,1,2, \ldots \text { is bounded }\right\} \text {. }
$$

We choose the initial point 0 as 0 is the only critical point of $P_{c}$ [20, p. 249].

Definition 2.4 Let $(X, d)$ be a metric space and $\mathrm{I}=[0,1]$. A mapping $\omega: X^{2} \times I \rightarrow X$ is said to be convex structure on $\mathrm{X}$, if for any $(x, y, \alpha) \in X^{2} \times I$ and $u \in X$, the following inequality holds:

$$
d(\omega(x, y, \alpha), u) \leq \alpha^{s} d(x, u)+(1-\alpha)^{s} d(y, u)
$$

The metric space $(X, d)$ with convex structure $\omega$ is called a convex metric space [30]. Moreover, a nonempty subset $\mathrm{C}$ of $\mathrm{X}$ is said to be convex if $\omega(x, y, \alpha) \in C$ for all $(x, y, \alpha) \in C^{2} \times I$.

Definition 2.5 Let $(X, d)$ be a metric space and $\mathrm{I}=[0,1]$ and $\left\{a_{n}\right\},\left\{b_{n}\right\},\left\{c_{n}\right\}$ are real sequences in $[0,1]$ with $a_{n}+b_{n}+c_{n}=1$. A mapping $\omega: X^{3} \times I^{3} \rightarrow X$ is said to be convex structure [38] on $\mathrm{X}$, if for any $\left(x, y, z, a_{n}, b_{n}, c_{n}\right) \in X^{3} \times I^{3}$ and $u \in X$, the following inequality holds:

$$
d\left(\omega\left(x, y, z, a_{n}, b_{n}, c_{n}\right), u\right) \leq\left(a_{n}\right)^{s} d(x, u)+\left(b_{n}\right)^{s} d(y, u)+\left(c_{n}\right)^{s} d(z, u)
$$

Moreover, a nonempty subset $\mathrm{C}$ of $\mathrm{X}$ is said to be convex if $\omega\left(x, y, z, a_{n}, b_{n}, c_{n}\right) \in C$ for all $\left(x, y, z, a_{n}, b_{n}, c_{n}\right) \in C^{3} \times I^{3}$.

Definition 2.6 Let $\mathrm{X}$ be a subset of real complex number and $T: X \rightarrow X$. Consider a sequence $\left\{z_{n}\right\}$ of iterates for initial point $z_{0} \in X$ such that

$$
\begin{gathered}
z_{n+1}=\left(1-\alpha_{n}\right) u_{n}+\alpha_{n} T\left(u_{n}\right) \\
u_{n}=\left(1-\beta_{n}\right) v_{n}+\beta_{n} T\left(v_{n}\right) \\
v_{n}=\left(1-\gamma_{n}\right) z_{n}+\gamma_{n} T\left(z_{n}\right)
\end{gathered}
$$


where $\mathrm{n}=0,1,2, \ldots, \alpha_{n}, \beta_{n}, \gamma_{n} \in[0,1]$ and $\left\{\alpha_{n}\right\},\left\{\beta_{n}\right\},\left\{\gamma_{n}\right\}$ are sequences of positive numbers. Then the sequence (2.1) of iterates is called SP orbit [29] having five tuples $\left(T, z_{0}, \alpha_{n}, \beta_{n}, \gamma_{n}\right)$.

Remark 2.7 The $S P$ Orbit reduces to:

1) The Thaiwan orbit [24] when $\gamma_{n}=0$, i.e.

$$
\begin{aligned}
z_{n+1} & =\left(1-\alpha_{n}\right) u_{n}+\alpha_{n} T\left(u_{n}\right) \\
u_{n} & =\left(1-\beta_{n}\right) z_{n}+\beta_{n} T\left(z_{n}\right)
\end{aligned}
$$

2) The Mann orbit ([18],[19]) when $\beta_{n}=\gamma_{n}=0$, i.e.

$$
z_{n+1}=\left(1-\alpha_{n}\right) z_{n}+\alpha_{n} T\left(z_{n}\right)
$$

3) The Picard orbit [20] when $\beta_{n}=\gamma_{n}=0$ and $\alpha_{n}=1$, i.e.

$$
z_{n+1}=T\left(z_{n}\right)
$$

\section{MAIN RESULTS}

In the generation and analysis of fractals, escape criterion plays a vital role. We need the following escape criterions for the quadratic, cubic and higher degree polynomials in SP orbit.

We assume that $z_{0}=z \in \mathbb{C}$ and $\alpha_{n}=\alpha, \beta_{n}=\beta, \gamma_{n}=\gamma$ then we can write SP iteration scheme with s-convexity in the following manner where $P_{c}\left(z_{n}\right)$ be a quadratic, cubic or nth degree polynomial:

$$
\begin{gathered}
z_{n+1}=\left(1-\alpha_{n}\right)^{s} u_{n}+\alpha^{s} P_{c}\left(u_{n}\right) \\
u_{n}=\left(1-\beta_{n}\right)^{s} v_{n}+\beta^{s} P_{c}\left(v_{n}\right) \\
v_{n}=\left(1-\gamma_{n}\right)^{S} z_{n}+\gamma^{s} P_{c}\left(z_{n}\right)
\end{gathered}
$$

where $0<\alpha, \beta, \gamma \leq 1$ and $0<s \leq 1$.

\section{A. Escape Criterions for Quadratic Polynomials}

Theorem 3.1 Suppose $|z| \geq|c|>\frac{2}{s \alpha},|z| \geq|c|>\frac{2}{s \beta}$ and $|z| \geq|c|>\frac{2}{s \gamma}$, where $0<\alpha, \beta, \gamma \leq 1$ and $c$ is a complex number. Define

$$
\begin{gathered}
z_{1}=(1-\alpha)^{s} u+\alpha^{s} P_{c}(u) \\
z_{2}=(1-\alpha)^{s} u_{1}+\alpha^{s} P_{c}\left(u_{1}\right) \\
--- \\
--- \\
--- \\
z_{n}=(1-\alpha)^{s} u_{n-1}+\alpha^{s} P_{c}\left(u_{n-1}\right)
\end{gathered}
$$

where $P_{c}(z)$ is a quadratic polynomial in terms of $\beta$ and $n=1,2,3, \ldots$, then $\left|z_{n}\right| \rightarrow \infty$ as $n \rightarrow \infty$.

Proof: Consider

$$
\begin{aligned}
&|v|=\left|(1-\gamma)^{s} z+\gamma^{s} P_{c}(z)\right|, \quad \text { for } P_{c}(z)=z^{2}+c, \\
&|v|=\left|(1-\gamma)^{s} z+\gamma^{s}\left(z^{2}+c\right)\right| \\
&=\left|(1-\gamma)^{s} z+(1-(1-\gamma))^{s}\left(z^{2}+c\right)\right|
\end{aligned}
$$

By binomial expansion upto linear terms of $\gamma$ and $(1-\gamma)$, we obtain

$$
\begin{aligned}
|v| & =\left|(1-s \gamma) z+(1-s(1-\gamma))\left(z^{2}+c\right)\right| \\
& =\left|(1-s \gamma) z+(1-s+s \gamma)\left(z^{2}+c\right)\right|
\end{aligned}
$$




$$
\begin{aligned}
& \geq\left|(1-s \gamma) z+s \gamma\left(z^{2}+c\right)\right|, \quad \text { because } 1-s+s \gamma \geq s \gamma \\
& \geq\left|s \gamma z^{2}+(1-s \gamma) z\right|-|s \gamma c| \\
& \geq\left|s \gamma z^{2}+(1-s \gamma) z\right|-|s \gamma z|, \quad(\because|z| \geq|c|) \\
& \geq\left|s \gamma z^{2}\right|-|(1-s \gamma) z|-|s \gamma z| \\
& \geq\left|s \gamma z^{2}\right|-|z|+|s \gamma z|-|s \gamma z| \\
& =\left|s \gamma z^{2}\right|-|z| \\
& \geq|z|(s \gamma|z|-1)
\end{aligned}
$$

i.e. $|v| \geq|z|(s \gamma|z|-1)$

Also for

$$
\begin{aligned}
|u| & =\left|(1-\beta)^{s} v+\beta^{s} P_{c}(v)\right| \\
& =\left|(1-\beta)^{s} v+\beta^{s}\left(v^{2}+c\right)\right| \\
& =\left|(1-\beta)^{s} v+(1-(1-\beta))^{s}\left(v^{2}+c\right)\right|
\end{aligned}
$$

By binomial expansion upto linear terms of $\beta$ and $(1-\beta)$, we obtain

$$
\begin{aligned}
|u| & =\left|(1-s \beta) v+(1-s(1-\beta))\left(v^{2}+c\right)\right| \\
& =\left|(1-s \beta) v+(1-s+s \beta)\left(v^{2}+c\right)\right| \\
& \geq\left|(1-s \beta) v+s \beta\left(v^{2}+c\right)\right|, \quad \text { because } 1-s+s \beta \geq s \beta
\end{aligned}
$$

Then using equation (3.1), we have

$$
|u| \geq|(1-s \beta)| z\left|(s \gamma|z|-1)+s \beta\left[\{|z|(s \gamma|z|-1)\}^{2}+c\right]\right|
$$

Since $|z| \geq(2 / s \gamma)$ implies $s \gamma|z|-1>1$ and $|z|^{2}(s \gamma|z|-1)^{2}>|z|^{2}$.

Then using this fact, equation (3.2) becomes

$$
\begin{aligned}
& |u| \geq|(1-s \beta)| z\left|+s \beta\left(|z|^{2}+c\right)\right| \\
& \geq\left.|s \beta| z\right|^{2}+(1-s \beta)|z||-| s \beta c \mid \\
& \geq\left.|s \beta| z\right|^{2}+(1-s \beta)|z|-|s \beta z|, \quad(\because|z| \geq|c|) \\
& \geq\left|s \beta z^{2}\right|-|(1-s \beta)| z||-|s \beta z| \\
& \geq|z|(s \beta|z|-1) \\
& \text { i. e. } \quad|u| \geq|z|(s \beta|z|-1) \text {. }
\end{aligned}
$$

Now for $z_{n}=(1-\alpha)^{s} u_{n-1}+\alpha^{s} P_{c}\left(u_{n-1}\right)$, we have

$$
\begin{aligned}
\left|z_{1}\right| & =\left|(1-\alpha)^{s} u+\alpha^{s} P_{c}(u)\right| \\
& =\left|(1-\alpha)^{s} u+\alpha^{s}\left(u^{2}+c\right)\right| \\
& =\left|(1-\alpha)^{s} u+(1-(1-\alpha))^{s}\left(u^{2}+c\right)\right|
\end{aligned}
$$

By binomial expansion upto linear terms of $\alpha$ and $(1-\alpha)$, we obtain 


$$
\begin{aligned}
\left|z_{1}\right| & =\left|(1-s \alpha) u+(1-s(1-\alpha))\left(u^{2}+c\right)\right| \\
& =\left|(1-s \alpha) u+(1-s+s \alpha)\left(u^{2}+c\right)\right| \\
& \geq\left|(1-s \alpha) u+s \alpha\left(u^{2}+c\right)\right|,
\end{aligned}
$$$$
\text { because } 1-s+s \alpha \geq s \alpha
$$

Then using equation (3.3), we have

$$
\left|z_{1}\right| \geq|(1-s \alpha)| z\left|(s \beta|z|-1)+s \alpha\left[\{|z|(s \beta|z|-1)\}^{2}+c\right]\right|
$$

Since $|z| \geq(2 / s \beta)$ implies $s \beta|z|-1>1$ and $|z|^{2}(s \beta|z|-1)^{2}>|z|^{2}$.

Then using this in equation (3.4), we have

$$
\begin{aligned}
\left|z_{1}\right| & \geq|(1-s \alpha)| z\left|+s \alpha\left(|z|^{2}+c\right)\right| \\
& \geq\left.|s \alpha| z\right|^{2}+(1-s \alpha)|z||-| s \alpha c \mid \\
& \geq\left.|s \alpha| z\right|^{2}+(1-s \alpha)|z||-| s \alpha z \mid \\
& \geq\left|s \alpha z^{2}\right|-|(1-s \alpha)| z||-|s \alpha z| \\
& \geq|z|(s \alpha|z|-1)
\end{aligned}
$$

Since $|z| \geq|c|>(2 / s \alpha),|z| \geq|c|>(2 / s \beta)$ and $|z| \geq|c|>(2 / s \gamma)$ exist. Therefore, we have $s \alpha|z|-1>1$. Hence there exists a $\lambda>0$ such that $s \alpha|z|-1>\lambda+1>1$. Consequently, we have

$$
\left|z_{1}\right|>(1+\lambda)|z|
$$

Particularly, $\left|z_{n}\right|>|z|$. So, repeating the same argument $\mathrm{n}$ times we obtain,

$$
\left|z_{n}\right|>(1+\lambda)^{n}|z| \text {. }
$$

Thus, the orbit of $z$ tends to infinity as $\mathrm{n}$ tends to infinity. Hence the result.

From the above theorem, we obtain the following corollaries:

Corollary 3.2 Assume that $|c|>2 / \mathrm{s} \alpha,|c|>2 / \mathrm{s} \beta$ and $|c|>2 / \mathrm{s} \gamma$, then the orbit $\operatorname{SP}\left(P_{c}, 0, s \alpha, s \beta, s \gamma\right)$ escapes to infinity.

In the above theorem, the escape criterion used, gives us a little more information. In the proof, we used the only fact that $|z|>|c|$ and $|c|>2 / \mathrm{s} \alpha,|c|>2 / \mathrm{s} \beta,|c|>2 / \mathrm{s} \gamma$. Hence we have the following refinement of the escape criterion:

Corollary 3.3 (Escape Criterion). Suppose $|z|>\max \{|c|, 2 / s \alpha, 2 / s \beta, 2 / s \gamma\}$, then $\quad\left|z_{n}\right|>(1+\lambda)^{n}|z| \quad$ and $\left|z_{n}\right| \rightarrow \infty$ as $n \rightarrow \infty$.

We notice that we may apply Corollary 3.3 to $\left|z_{k}\right|$ for some $k \geq 0$ to have the following result.

Corollary 3.4 Suppose $\left|z_{k}\right|>\max \{|c|, 2 / s \alpha, 2 / s \beta, 2 / s \gamma\}$, for some $k \geq 0$, then we have,

$$
\left|z_{k+1}\right|>(1+\lambda)^{n}\left|z_{k}\right| \text { and }\left|z_{n}\right| \rightarrow \infty \text { as } n \rightarrow \infty \text {. }
$$

Using this corollary, we obtain an algorithm for computing the connected Julia sets of quadratic $P_{c}$, for any complex number ' $c$ '. For given any point ' $z$ ' satisfying $|z| \leq|c|$, we obtain the orbit of ' $z$ '. If, for some ' $n$ ', $\left|z_{n}\right|$ lies outside the circle of radius $\max \{|c|, 2 / s \alpha, 2 / s \beta, 2 / s \gamma\}$, then we observe that the orbit escapes to infinity, which means that ' $z$ ' does not lie in the connected Julia set. If $\left|z_{n}\right|$ never exceeds this bound, then by definition ' $z$ ' lies in the connected Julia set. 


\section{B. Escape Criterions for Cubic Polynomials}

Now, we prove the following theorem for a cubic polynomial $P_{a, b}(z)=z^{3}+a z+b$, where $\mathrm{a}, \mathrm{b}$ are complex numbers, as this polynomial is equivalent to all other cubic polynomials.

Theorem 3.5 Suppose that $|z| \geq|b|>(|a|+2 / s \alpha)^{1 / 2}, \quad|z| \geq|b|>(|a|+2 / s \beta)^{1 / 2}$ and $|z| \geq|b|>(|a|+2 / s \gamma)^{1 / 2}$, where $0<\alpha, \beta, \gamma \leq 1$, and $a, b$ are in the complex plane. Define

$$
\begin{aligned}
& z_{1}=(1-\alpha)^{S} u+\alpha^{S} P_{a, b}(u) \\
& z_{2}=(1-\alpha)^{S} u_{1}+\alpha^{S} P_{a, b}\left(u_{1}\right) \\
& --- \\
& --- \\
& -- \\
& z_{n}=(1-\alpha)^{s} u_{n-1}+\alpha^{S} P_{a, b}\left(u_{n-1}\right), n=1,2,3 \ldots
\end{aligned}
$$

where $P_{a, b}(u)$ is a cubic polynomial in terms of $\beta$, then $\left|z_{n}\right| \rightarrow \infty$ as $n \rightarrow \infty$.

Proof: Consider

$$
\begin{aligned}
|v| & =\left|(1-\gamma)^{s} z+\gamma^{s} P_{a, b}(z)\right|, \quad \text { for } P_{a, b}(z)=z^{3}+a z+b \\
|v| & =\left|(1-\gamma)^{s} z+\gamma^{s}\left(z^{3}+a z+b\right)\right| \\
& =\left|(1-\gamma)^{s} z+(1-(1-\gamma))^{s}\left(z^{3}+a z+b\right)\right|
\end{aligned}
$$

By binomial expansion upto linear terms of $\gamma$ and $(1-\gamma)$, we obtain

$$
\begin{aligned}
|v| & =\left|(1-s \gamma) z+(1-s(1-\gamma))\left(z^{3}+a z+b\right)\right| \\
& =\left|(1-s \gamma) z+(1-s+s \gamma)\left(z^{3}+a z+b\right)\right| \\
& \geq\left|(1-s \gamma) z+s \gamma\left(z^{3}+a z+b\right)\right|, \quad \text { because } 1-s+s \gamma \geq s \gamma \\
& \geq\left|s \gamma z^{3}+s \gamma a z+s \gamma b+(1-s \gamma) z\right| \\
& \geq\left|s \gamma z^{3}+s \gamma a z+(1-s \gamma) z\right|-|s \gamma z|, \\
& \geq\left|s \gamma z^{3}\right|-|s \gamma a z|-|(1-s \gamma) z|-|s \gamma z| \\
& \geq\left|s \gamma z^{3}\right|-|s \gamma a z|-|z| \\
& \geq|z|\left(s \gamma\left(|z|^{2}-|a|\right)-1\right) . \\
\text { i.e. } \quad v & \geq|z|\left(s \gamma\left(|z|^{2}-|a|\right)-1\right)
\end{aligned}
$$

Also for

$$
\begin{aligned}
|u| & =\left|(1-\beta)^{s} v+\beta^{s} P_{a, b}(v)\right| \\
& =\left|(1-\beta)^{s} v+\beta^{s}\left(v^{3}+a v+b\right)\right| \\
& =\left|(1-\beta)^{s} v+(1-(1-\beta))^{s}\left(v^{3}+a v+b\right)\right|
\end{aligned}
$$

By binomial expansion upto linear terms of $\beta$ and $(1-\beta)$, we obtain

$|u|=\left|(1-s \beta) v+(1-s(1-\beta))\left(v^{3}+a v+b\right)\right|$ 


$$
\begin{aligned}
& =\left|(1-s \beta) v+(1-s+s \beta)\left(v^{3}+a v+b\right)\right| \\
& \geq\left|(1-s \beta) v+s \beta\left(v^{3}+a v+b\right)\right|, \quad \text { because } 1-s+s \beta \geq s \beta
\end{aligned}
$$

Then using equation (3.5), we have

$$
|u| \geq|(1-s \beta)| z\left|\left\{s \gamma\left(|z|^{2}-|a|\right)-1\right\}+s \beta\left[\left\{|z|\left(s \gamma\left(|z|^{2}-|a|\right)-1\right)\right\}^{3}+a|z|\left(s \gamma\left(|z|^{2}-|a|\right)-1\right)+b\right]\right|
$$

Since $|z| \geq(|a|+2 / s \gamma)^{1 / 2}$ which implies $s \gamma\left(|z|^{2}-|a|\right)-1>1$ and $|z|^{3}\left(s \gamma\left(|z|^{2}-|a|\right)-1\right)^{3}>|z|^{3}$.

Then using this fact in equation (3.6), we have

$$
\begin{aligned}
& \qquad \begin{aligned}
|u| & \geq|(1-s \beta)| z\left|+s \beta\left(|z|^{3}+a|z|+b\right)\right| \\
& \geq\left.|s \beta| z\right|^{3}+s \beta a|z|+(1-s \beta)|z||-| s \beta b \mid \\
& \geq\left.|s \beta| z\right|^{3}|-| s \beta a z|-|(1-s \beta)|z||-| s \beta z \mid, \quad(\because|z| \geq|\mathrm{b}|) \\
& \geq\left|s \beta z^{3}\right|-|s \beta a z|-|z| \\
& \geq|z|\left(s \beta\left(|z|^{2}-|a|\right)-1\right)
\end{aligned} \\
& \text { i. e. }|u| \geq|z|\left(s \beta\left(|z|^{2}-|a|\right)-1\right) .
\end{aligned}
$$

Now for $z_{n}=(1-\alpha)^{s} u_{n-1}+\alpha^{s} P_{a, b}\left(u_{n-1}\right)$, we have

$$
\begin{aligned}
\left|z_{1}\right| & =\left|(1-\alpha)^{s} u+\alpha^{s} P_{a, b}(u)\right| \\
& =\left|(1-\alpha)^{s} u+\alpha^{s}\left(u^{3}+a u+b\right)\right| \\
& =\left|(1-\alpha)^{s} u+(1-(1-\alpha))^{s}\left(u^{3}+a u+b\right)\right|
\end{aligned}
$$

By binomial expansion upto linear terms of $\alpha$ and $(1-\alpha)$, we obtain

$$
\begin{aligned}
\left|z_{1}\right| & =\left|(1-s \alpha) u+(1-s(1-\alpha))\left(u^{3}+a u+b\right)\right| \\
& =\left|(1-s \alpha) u+(1-s+s \alpha)\left(u^{3}+a u+b\right)\right| \\
& \geq\left|(1-s \alpha) u+s \alpha\left(u^{3}+a u+b\right)\right|,
\end{aligned}
$$$$
\text { because } 1-s+s \alpha \geq s \alpha
$$

Then using equation (3.7), we have

$$
\left|z_{1}\right| \geq|(1-s \alpha)| z\left|\left\{s \beta\left(|z|^{2}-|a|\right)-1\right\}+s \alpha\left[\left\{|z|\left(s \beta\left(|z|^{2}-|a|\right)-1\right)\right\}^{3}+a|z|\left(s \beta\left(|z|^{2}-|a|\right)-1\right)+b\right]\right|
$$

Since $|z| \geq(|a|+2 / s \beta)^{1 / 2}$ implies $s \beta\left(|z|^{2}-|a|\right)-1>1$ and $|z|^{3}\left(s \beta\left(|z|^{2}-|a|\right)-1\right)^{3}>|z|^{3}$.

By using this fact, equation (3.8) reduces to

$$
\begin{aligned}
\left|z_{1}\right| & \geq|(1-s \alpha)| z\left|+s \alpha\left(|z|^{3}+a|z|+b\right)\right| \\
& \geq\left.|s \alpha| z\right|^{3}+s \alpha a|z|+(1-s \alpha)|z||-| s \alpha b \mid \\
& \geq\left.|s \alpha| z\right|^{3}|-| s \alpha a|z||-|(1-s \alpha)|z||-| s \alpha z \mid \\
& \geq\left|s \alpha z^{3}\right|-|s \alpha a| z||-|z|
\end{aligned}
$$




$$
\geq|z|\left(s \alpha\left(|z|^{2}-|a|\right)-1\right)
$$

Since $|z| \geq|b|>(|a|+2 / s \alpha)^{1 / 2},|z| \geq|b|>(|a|+2 / s \beta)^{1 / 2}$ and $|z| \geq|b|>(|a|+2 / s \gamma)^{1 / 2}$ exist. Therefore, we have $s \alpha\left(|z|^{2}-|a|\right)-1>1$. Hence, there exists a $\lambda>0$ such that $s \alpha\left(|z|^{2}-|a|\right)-1>\lambda+1>1$. Consequently, we have

$$
\left|z_{1}\right|>(1+\lambda)|z|
$$

Particularly, $\left|z_{n}\right|>|z|$. So, repeating the same argument $n$ times we obtain,

$$
\left|z_{n}\right|>(1+\lambda)^{n}|z|
$$

Thus, the orbit of $z$ tends to infinity as $n$ tends to infinity. Hence the result.

Using above result, we obtain the following corollaries:

Corollary 3.6 (Escape Criterion): Let $P_{a, b}(z)=z^{3}+a z+b$, where $a, b$ are any complex numbers. If $|z|>\max \left\{|b|,(|a|+2 / s \alpha)^{1 / 2},(|a|+2 / s \beta)^{1 / 2},(|a|+2 / s \gamma)^{1 / 2}\right\}$, then $\left|z_{n}\right| \rightarrow \infty$ as $n \rightarrow \infty$. This gives the escape criterion for a cubic polynomial.

Corollary 3.7 For some $k \geq 0$, let us suppose $\left|z_{k}\right|>\max \left\{|b|,(|a|+2 / s \alpha)^{1 / 2},(|a|+2 / s \beta)^{1 / 2},(|a|+2 / s \gamma)^{1 / 2}\right\}$. Then $\left|z_{k+1}\right|>\lambda\left|z_{k}\right|$ and $\left|z_{n}\right| \rightarrow \infty$ as $n \rightarrow \infty$.

We find that Corollary 3.7 gives the algorithm to generate Julia sets for cubic polynomial $P_{a, b}(z)$.

\section{A General Escape Criterion}

Now, we obtain a general escape criterion for polynomials of the form $G_{c}(z)=z^{n}+c$

Theorem 3.8 For a general function $G_{c}(z)=z^{n}+c, n=1,2,3 \ldots$, where $0<\alpha, \beta, \gamma \leq 1$, and $c$ is a complex number. Define

$$
\begin{aligned}
& z_{1}=(1-\alpha)^{s} u+\alpha^{s} G_{c}(u) \\
& z_{2}=(1-\alpha)^{s} u_{1}+\alpha^{s} G_{c}\left(u_{1}\right) \\
& \quad-- \\
& \quad-- \\
& \quad-- \\
& z_{n}=(1-\alpha)^{s} u_{n-1}+\alpha^{s} G_{c}\left(u_{n-1}\right), \quad n=1,2,3,4, \ldots
\end{aligned}
$$

Then, the general escape criterion is $\max \left\{|c|,(2 / s \alpha)^{1 / n-1},(2 / s \beta)^{1 / n-1},(2 / s \gamma)^{1 / n-1}\right\}$.

Proof: For proving the theorem, we shall use the method of induction.

For $n=1$, we have $G_{c}(z)=z+c$, and this implies

$$
|z|>\max \{|c|, 0,0,0\} \text {. }
$$

For $n=2$, we have $G_{c}(z)=z^{2}+c$, so by theorem 3.1 , the escape criterion is

$$
|z|>\max \{|c|, 2 / s \alpha, 2 / s \beta, 2 / s \gamma\} \text {. }
$$

Similarly, for $n=3$, we get $G_{c}(z)=z^{3}+c$. Then the escape criterion from the theorem 3.5 is

Hence, the theorem is true for $n=1,2,3$.

$$
|z|>\max \left\{|c|,(2 / s \alpha)^{1 / 2},(2 / s \beta)^{1 / 2},(2 / s \gamma)^{1 / 2}\right\} .
$$

Now, suppose that theorem is true for any $\mathrm{n}$. We prove that the result is true for $\mathrm{n}+1$. Let $G_{c}(z)=z^{n+1}+c$ and $|z| \geq|c|>(2 / s \alpha)^{1 / n},|z| \geq|c|>(2 / s \beta)^{1 / n}$ and $|z| \geq|c|>(2 / s \gamma)^{1 / n}$.

Then, consider

$$
\begin{aligned}
|v| & =\left|(1-\gamma)^{s} z+\gamma^{s} G_{c}(z)\right|, \quad \text { where } G_{c}(z)=z^{n+1}+c \\
& =\left|(1-\gamma)^{s} z+\gamma^{s}\left(z^{n+1}+c\right)\right|
\end{aligned}
$$




$$
=\left|(1-\gamma)^{s} z+(1-(1-\gamma))^{s}\left(z^{n+1}+c\right)\right|
$$

By binomial expansion upto linear terms of $\gamma$ and $(1-\gamma)$, we obtain

$$
\begin{aligned}
|v| & =\left|(1-s \gamma) z+(1-s(1-\gamma))\left(z^{n+1}+c\right)\right| \\
& =\left|(1-s \gamma) z+(1-s+s \gamma)\left(z^{n+1}+c\right)\right| \\
& \geq\left|(1-s \gamma) z+s \gamma\left(z^{n+1}+c\right)\right|, \quad \text { because } 1-s+s \gamma \geq s \gamma \\
& \geq\left|s \gamma z^{n+1}+(1-s \gamma) z\right|-|s \gamma c| \\
& \geq\left|s \gamma z^{n+1}+(1-s \gamma) z\right|-|s \gamma z|, \\
& \geq\left|s \gamma z^{n+1}\right|-|(1-s \gamma) z|-|s \gamma z| \\
& \geq\left|s \gamma z^{n+1}\right|-|z|+|s \gamma z|-|s \gamma z| \\
& =\left|s \gamma z^{n+1}\right|-|z| \\
& \geq|z|\left(s \gamma|z|^{n}-1\right), \\
\text { i.e } \quad|v| & \geq|z|\left(s \gamma|z|^{n}-1\right)
\end{aligned}
$$

Also for

$$
\begin{aligned}
|u| & =\left|(1-\beta)^{s} v+\beta^{s} G_{c}(v)\right|, \\
& =\left|(1-\beta)^{s} v+\beta^{s}\left(v^{n+1}+c\right)\right| \\
& =\left|(1-\beta)^{s} v+(1-(1-\beta))^{s}\left(v^{n+1}+c\right)\right|
\end{aligned}
$$

By binomial expansion upto linear terms of $\beta$ and $(1-\beta)$, we obtain

$$
\begin{aligned}
|u| & =\left|(1-s \beta) v+(1-s(1-\beta))\left(v^{n+1}+c\right)\right| \\
& =\left|(1-s \beta) v+(1-s+s \beta)\left(v^{n+1}+c\right)\right| \\
& \geq\left|(1-s \beta) v+s \beta\left(v^{n+1}+c\right)\right|, \quad \text { because } 1-s+s \beta \geq s \beta
\end{aligned}
$$

Then using equation (3.9), we have

$$
|u| \geq|(1-s \beta)| z\left|\left(s \gamma|z|^{n}-1\right)+s \beta\left[\left\{|z|\left(s \gamma|z|^{n}-1\right)\right\}^{n+1}+c\right]\right|
$$

Since $|z|>(2 / s \gamma)^{1 / n}$ implies $s \gamma|z|^{n}-1>1$ and $|z|^{n+1}\left(s \gamma|z|^{n}-1\right)^{n+1}>|z|^{n+1}$.

Using this fact, equation (3.10) becomes

$$
\begin{aligned}
|u| & \geq|(1-s \beta)| z\left|+s \beta\left(|z|^{n+1}+c\right)\right| \\
& \geq\left.|s \beta| z\right|^{n+1}+(1-s \beta)|z|-|s \beta c| \\
& \geq\left.|s \beta| z\right|^{n+1}+(1-s \beta)|z|-|s \beta z|, \quad(\because|z| \geq|c|) \\
& \geq\left|s \beta z^{n+1}\right|-|(1-s \beta)| z||-|s \beta z| \\
& \geq|z|\left(s \beta|z|^{n}-1\right)
\end{aligned}
$$




$$
\text { i. e., } \quad|u| \geq|z|\left(s \beta|z|^{n}-1\right) .
$$

Now for $z_{n}=(1-\alpha)^{s} u_{n-1}+\alpha^{s} G_{c}\left(u_{n-1}\right)$, we have

$$
\begin{aligned}
\left|z_{1}\right| & =\left|(1-\alpha)^{s} u+\alpha^{s} G_{c}(u)\right| \\
& =\left|(1-\alpha)^{s} u+\alpha^{s}\left(u^{n+1}+c\right)\right| \\
& =\left|(1-\alpha)^{s} u+(1-(1-\alpha))^{s}\left(u^{n+1}+c\right)\right|
\end{aligned}
$$

By binomial expansion upto linear terms of $\alpha$ and $(1-\alpha)$, we obtain

$$
\begin{aligned}
\left|z_{1}\right| & =\left|(1-s \alpha) u+(1-s(1-\alpha))\left(u^{n+1}+c\right)\right| \\
& =\left|(1-s \alpha) u+(1-s+s \alpha)\left(u^{n+1}+c\right)\right| \\
& \geq\left|(1-s \alpha) u+s \alpha\left(u^{n+1}+c\right)\right|, \quad \text { because } 1-s+s \alpha \geq s \alpha
\end{aligned}
$$

Then using equation (3.11), we obtain

$$
\left|z_{1}\right| \geq|(1-s \alpha)| z\left|\left(s \beta|z|^{n}-1\right)+s \alpha\left[\left\{|z|\left(s \beta|z|^{n}-1\right)\right\}^{n+1}+c\right]\right|
$$

Since $|z|>(2 / s \beta)^{1 / n}$ which implies $s \beta|z|^{n}-1>1$ and $|z|^{n+1}\left(s \beta|z|^{n}-1\right)^{n+1}>|z|^{n+1}$.

Using this in equation (3.12), we have

$$
\begin{aligned}
\left|z_{1}\right| & \geq|(1-s \alpha)| z\left|+s \alpha\left(|z|^{n+1}+c\right)\right| \\
& \geq\left.|s \alpha| z\right|^{n+1}+(1-s \alpha)|z||-| s \alpha c \mid \\
& \geq\left.|s \alpha| z\right|^{n+1}+(1-s \alpha)|z||-| s \alpha z \mid \\
& \geq\left|s \alpha z^{n+1}\right|-|(1-s \alpha)| z||-|s \alpha z| \\
& \geq|z|\left(s \alpha|z|^{n}-1\right)
\end{aligned}
$$

Since $|z| \geq|c|>(2 / s \alpha)^{1 / n},|z| \geq|c|>(2 / s \beta)^{1 / n}$ and $|z| \geq|c|>(2 / s \gamma)^{1 / n}$ exist. Therefore, we have $s \alpha|z|^{n}-1>1$. Hence there exists a $\lambda>0$ such that $s \alpha|z|-1>\lambda+1>1$.

In particular, $\quad\left|z_{1}\right|>(1+\lambda)|z|$

$$
\left|z_{n}\right|>(1+\lambda)^{n}|z|
$$

Hence,

$$
\left|z_{n}\right| \rightarrow \infty \text { as } n \rightarrow \infty \text {. }
$$

This completes the proof.

Corollary 3.9 Suppose $|c|>(2 / s \alpha)^{1 / n-1}, \quad|c|>(2 / s \beta)^{1 / n-1}$ and $|c|>(2 / s \gamma)^{1 / n-1}$ exists. Then the orbit $S P\left(G_{c}, 0, s \alpha, s \beta, s \gamma\right)$ escapes to infinity.

Corollary 3.10 (Escape Criterion). Let us assume that for some $\mathrm{k} \geq 0$, $\left|z_{k}\right|>\max \left\{|c|,(2 / s \alpha)^{1 / k-1},(2 / s \beta)^{1 / k-1},(2 / s \gamma)^{1 / k-1}\right\}$, then $\left|z_{k}\right|>\lambda\left|z_{k-1}\right|$ and $\left|z_{n}\right| \rightarrow \infty$ as $n \rightarrow \infty$.

This corollary gives an algorithm to generate Julia sets for the functions of the type $G_{c}(z)=z^{n}+c, \mathrm{n}=2,3 \ldots$ 


\section{GENERATION OF MANDELBROT SETS AND JULIA SETS}

In this section, using Mathematica 9.0, we generate some Mandelbrot sets and Julia sets for quadratic and cubic polynomials with s - convexity. After changing the value of convex parameter s and keeping the values of other parameters same, we have the following observations:

\subsection{Mandelbrot sets for quadratic polynomial $P_{c}(z)=z^{2}+c$}

(i) We obtain quadratic Mandelbrot sets via SP orbit with s-convexity see Figs. 1-6.

(ii) We use the maximum number of iterations 15 and grid length $[-3.6,1.0] \times[-2.5,2.5]$.

(iii) We observe that as we increase the value of $\mathrm{s}$, the range of Mandelbrot increase and it becomes fattier.

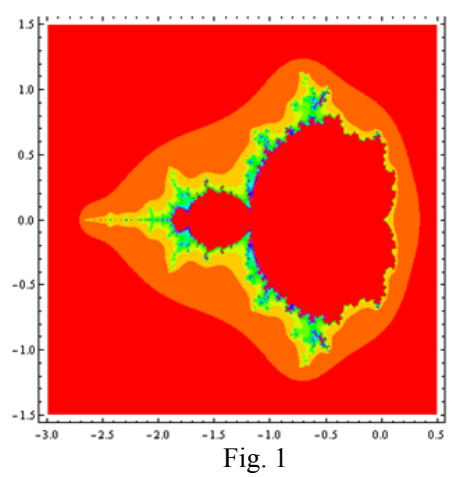

Quadratic Mandelbrot sets for $\alpha=\beta=\gamma=0.5, s=0.1$

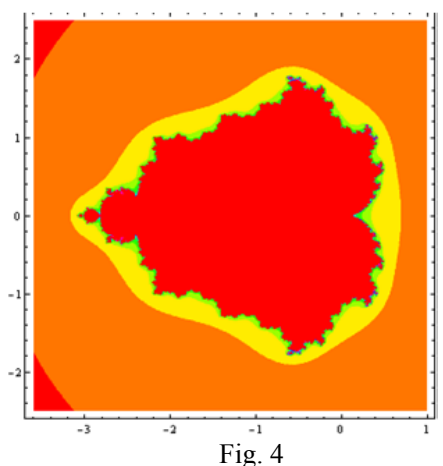

Quadratic Mandelbrot sets for $\alpha=\beta=\gamma=0.5, s=0.7$

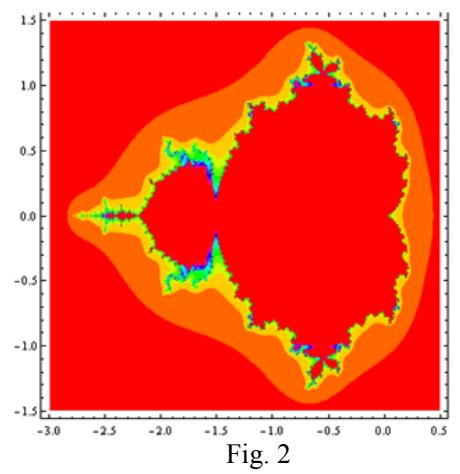

Quadratic Mandelbrot sets for $\alpha=\beta=\gamma=0.5, s=0.3$

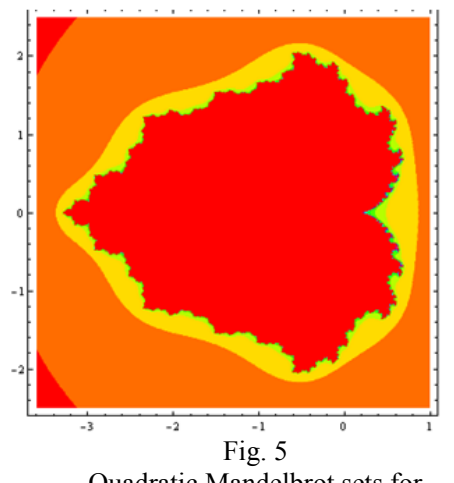

Quadratic Mandelbrot sets for $\alpha=\beta=\gamma=0.5, s=0.9$

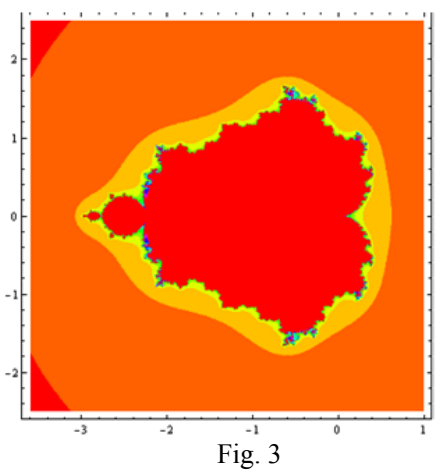

Quadratic Mandelbrot sets for $\alpha=\beta=\gamma=0.5, s=0.6$

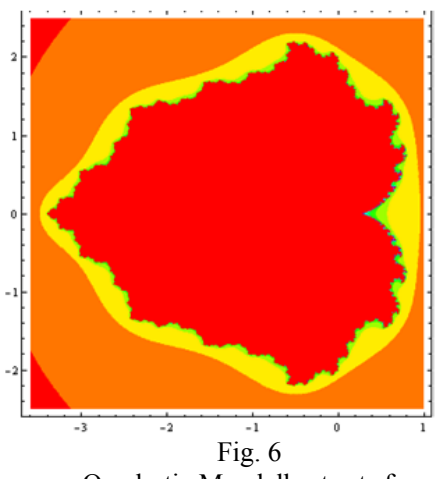

Quadratic Mandelbrot sets for $\alpha=\beta=\gamma=0.5, s=1$

4.2 Mandelbrot sets for cubic polynomial $\boldsymbol{P}_{0, c}(\mathbf{z})=\mathbf{z}^{3}+\boldsymbol{C}$

(i) In Figs. 7-12, we present the cubic Mandelbrot sets in SP orbit with s-convexity.

(ii) Mandelbrot sets are obtained by using maximum number of 15 iterations and grid length is $[-3.8,3.8] \times[-5.2,5.2]$.

(iii) We observe that as we increase the value of $\mathrm{s}$, the number of antennas of Mandelbrot sets is decreased. 


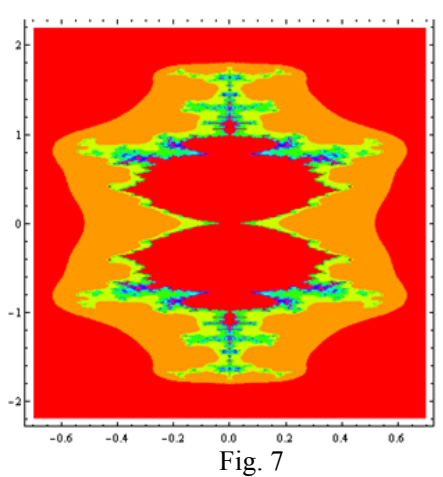

Cubic Mandelbrot sets for $\alpha=\beta=\gamma=0.115, s=0.1$

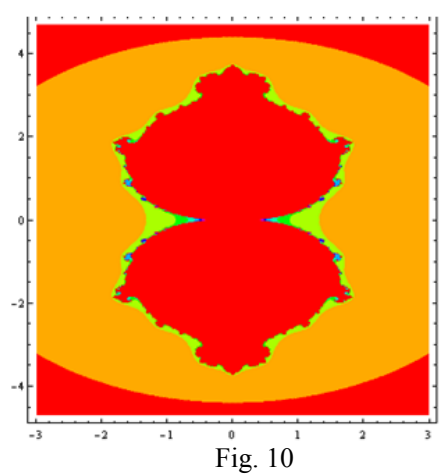

Cubic Mandelbrot sets for $\alpha=\beta=\gamma=0.115, s=0.8$

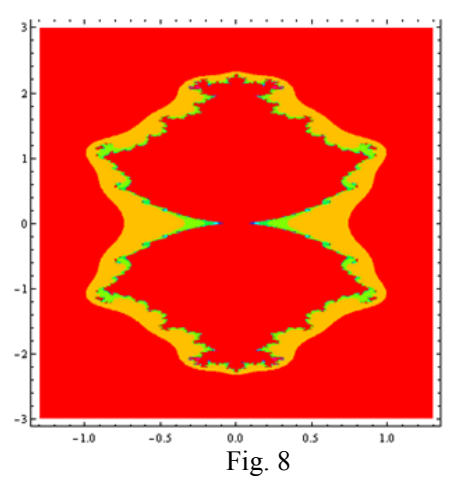

Cubic Mandelbrot sets for $\alpha=\beta=\gamma=0.115, s=0.4$

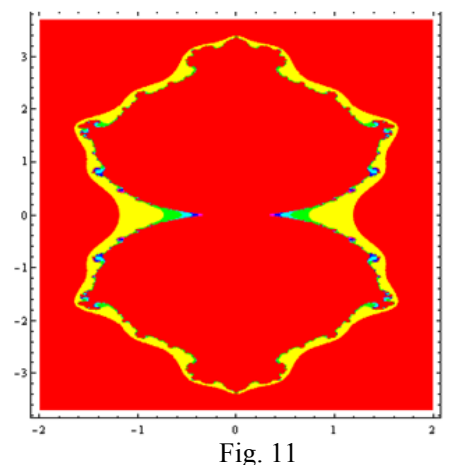

Cubic Mandelbrot sets for $\alpha=\beta=\gamma=0.115, s=0.9$

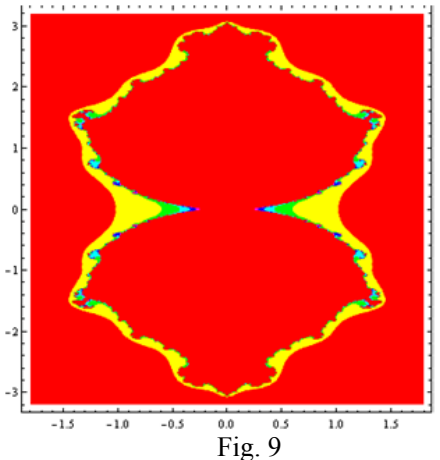

Cubic Mandelbrot sets for $\alpha=\beta=\gamma=0.115, s=0.7$

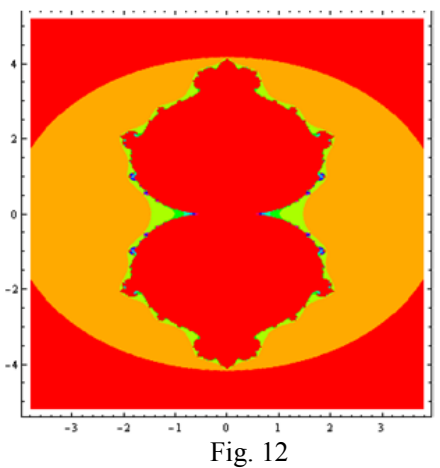

Cubic Mandelbrot sets for $\alpha=\beta=\gamma=0.115, s=1$

\subsection{Julia sets for quadratic and cubic polynomials:}

(i) We compute quadratic Julia sets in SP orbit with s-convexity (Figs. 13-18) and cubic Julia sets (Figs. 19-24).

(ii) We use the maximum number of 15 iterations for obtaining these Julia sets.

(iii) The maximum grid length for quadratic Julia sets is $[-1.8,1.8] \times[-1.2,1.2]$ and for cubic Julia sets is $[-1.3,1.3] \times[-2,2]$.

(iv) We observe that the boundary of quadratic Julia set becomes clearer as we increase the value of $\mathrm{s}$ [see figs. 13-18].

(v) Also, the structure of Cubic Julia sets varies with the variation of s [see figs. 19-24].
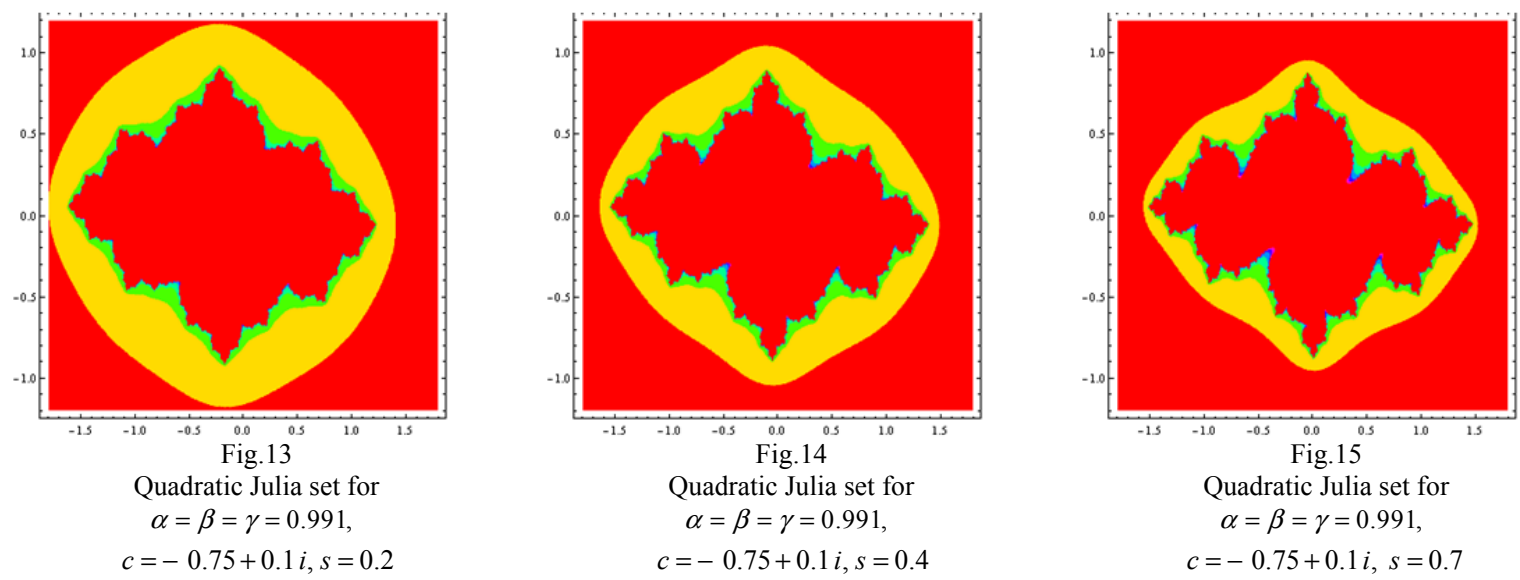


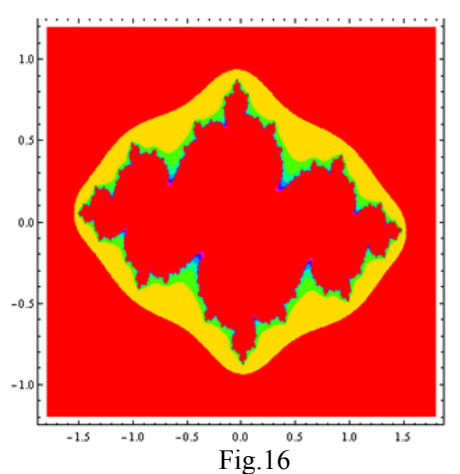

Quadratic Julia set for $\alpha=\beta=\gamma=0.991$, $c=-0.75+0.1 i, s=0.8$

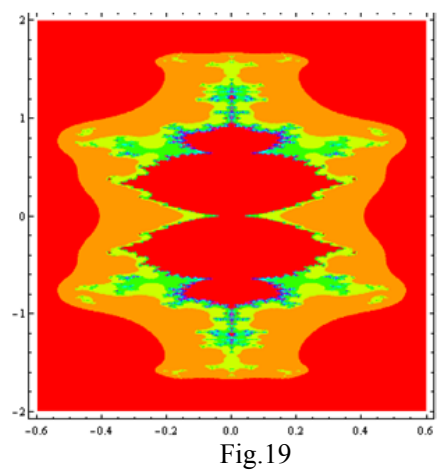

Cubic Julia set for $\alpha=\beta=\gamma=0.5$, $c=-0.7+0.4 i, s=0.1$

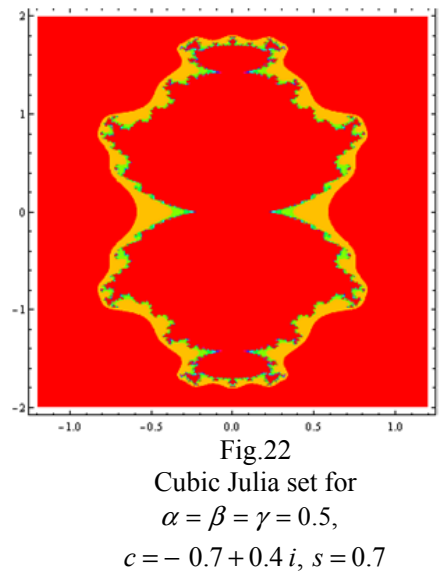

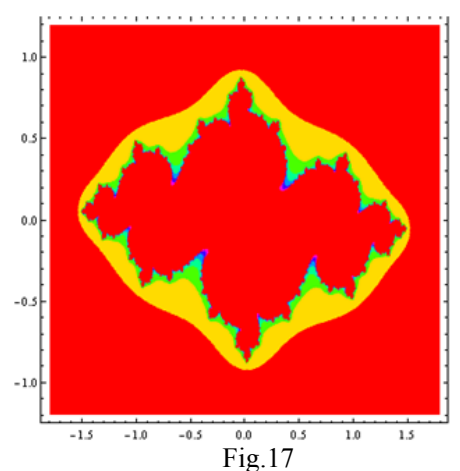

Quadratic Julia set for $\alpha=\beta=\gamma=0.991$,

$c=-0.75+0.1 i, s=0.9$

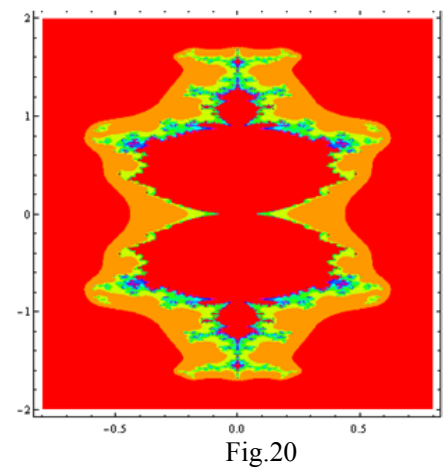

Cubic Julia set for $\alpha=\beta=\gamma=0.5$,

$c=-0.7+0.4 i, s=0.3$

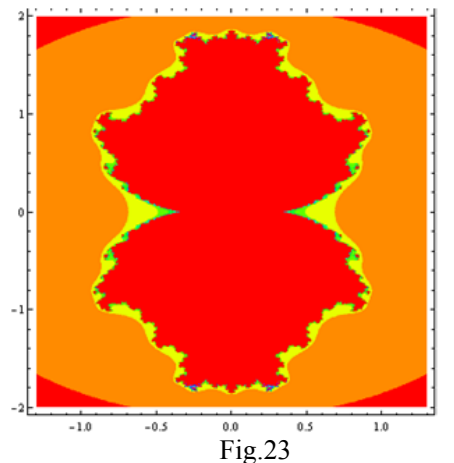

Cubic Julia set for $\alpha=\beta=\gamma=0.5$,

$c=-0.7+0.4 i, s=0.9$

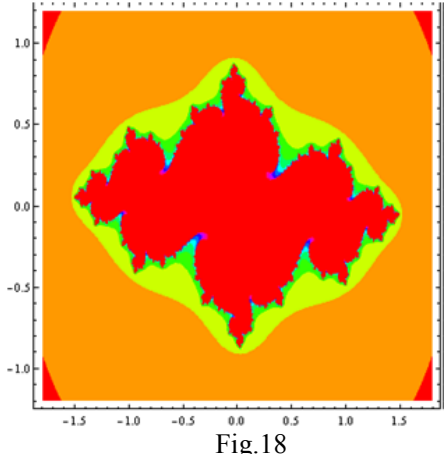

Quadratic Julia set for $\alpha=\beta=\gamma=0.991$,

$c=-0.75+0.1 i, s=1$

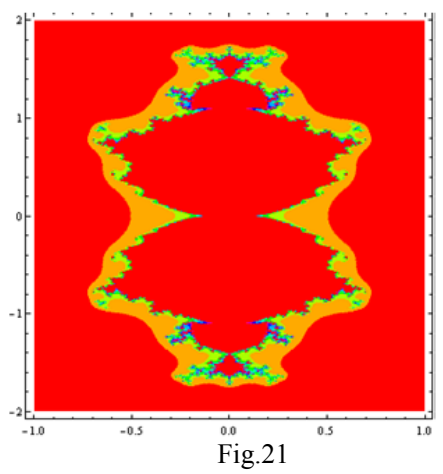

Cubic Julia set for $\alpha=\beta=\gamma=0.5$,

$c=-0.7+0.4 i, s=0.5$

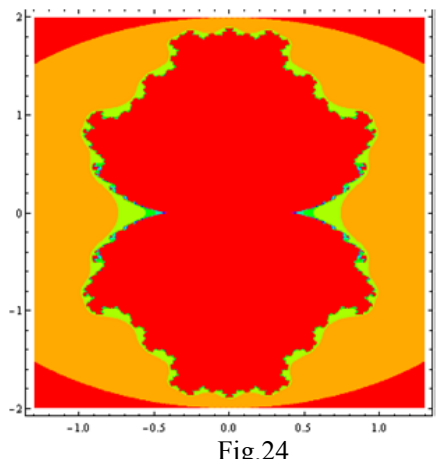

Cubic Julia set for $\alpha=\beta=\gamma=0.5$, $c=-0.7+0.4 i, s=1$

\section{CONCLUSION}

In this paper, we have obtained some fixed point results for generating fractals using SP- iteration with a convex structure. We have established the new escape criterion for complex quadratic, cubic and nth degree polynomials. The amazing thing which we notice that the Mandelbrot set varies with the variation of s, while this variation slightly affects the Julia set. Our results are the extension of existing results in the relative literature, as the SP-iteration reduces to Thaiwan's iteration, Mann's iteration and Picard's iteration which are examples of three-step, two-step and one-step iterative procedures respectively.

\section{ACKNOWLEDGMENT}

This work is supported by the University Grants Commission of India (Grant Nos. F.25-1/2014-15(BSR)/7392/2012(BSR) and F.173/2008 (SA-1)). 


\section{REFERENCES}

[1] A. Douady and J. H. Hubbard, tude dynamique des plynomes complexes, Pub-lications Mathematiques dOrsay 84 02; Universit de Paris-Sud, 1984.

[2] A. Epstein and M. Yampolsky, Geography of the cubic connectedness locus: intertwining surgery, Ann. Sci. cole Norm. Sup. 4(32)(2)(1999), 151-185.

[3] B. B. Mandelbrot, The Fractal Geometry of Nature, W. H. Freeman, New York, NY, USA, 1982.

[4] B. Branner and J. H. Hubbard, The Iteration of Cubic Polynomials Part I: The global topology of parameter space, Acta Math.,(160)(3-4)(1988), 143-206.

[5] B. Branner and J. H. Hubbard, The Iteration of Cubic Polynomials Part II: Patterns and parapatterns, Acta Math.,(169)(3-4)(1992), 229325.

[6] B. Ojha and M. K. Mishra, An application of xed point theorem for s-convex function, International Journal of Engineering Science and Technology, vol. 2, no. 8, (2010),3371-3375.

[7] D. Rochon, A Generalized Mandelbrot set for bicomplex numbers, Fractals,(8)(4)(2000), 355-368.

[8] F. Bernstein and G. Doetsch, Zur Theorie der konvexen Funktionen, Math. Ann. 76; (1915); 514-526.

[9] G. Julia, Sur literation des functions rationnelles, Journal de Mathematiques Pures et Appliquees, vol. 8; (1918), pp. $737-747$.

[10] H. Hudzik and L. Maligranda, Some remarks on s-convex functions, Aequa-tiones Mathematicae, vol. 48, no. 1; (1994), pp. 100111.

[11] H. O. Peitgen, H. Jurgens and D. Saupe, Chaos and Fractals, Springer-Verlag, New York, Inc., 1992.

[12] M. Alomari and M. Darus, Hadamard-type inequalities for s-convex functions, International Mathematical Forum, vol. 3, no.40,(2008), pp. 1965-1975.

[13] M. Alomari, M. Darus, On Co-ordinated s-convex functions, International Mathematical Forum, 3(40); (2008), $1977-1989$.

[14] M. Frame and J. Robertson, A generalized mandelbrot set and the role of critical points, Computer \& Graphics, (16)(1)(1992), 3540.

[15] M. K. Mishra , D. B. Ojha and D. Sharma, Fixed Point Results In Tricorn \& Multicorns of Ishikawa Iteration and s-Convexity, International Journal of Ad-vanced Engineering Sciences and Technologies, Vol No. 2, Issue No. 2; (2011), $156-159$.

[16] M. Kumari, Ashish and R. Chugh, New Julia and Mandelbrot sets for a new faster iterative process, International Journal of Pure and Applied Mathematics, (107)(1)(2016); 161-177.

[17] M. Rani , Cubic superior Julia sets, in: Proc. European Computing Confer-ence, 2011,80-84.

[18] M. Rani and V. Kumar, Superior Mandelbrot set, J. Korean Soc. Math. Edu. Res. Ser. D, (8)(4)(2004), $279-291$.

[19] M. Rani and V. Kumar, Superior Julia set, J. Korean Soc. Math. Edu. Res. Ser. D, (8)(4)(2004), 261-277.

[20] R. L. Devaney, A First Course in Chaotic Dynamical Systems: Theory and Experiment, Addison-Wesley, 1992.

[21] R. Rana, Y.S. Chauhan, A. Negi : Non linear dynamics of Ishikawa iterates. Int. J. Comput. Appl. 7, 13(2010).

[22] S. M. Kang, W. Nazeer, M. Tanveer and A. A. Shahid, New Fixed Point Results for Fractal Generation in Jungck Noor Orbit with s-Convexity, Journal of Function Spaces, Volume 2015, (2015), 7 pages.

[23] S. S. Liaw, The parameter spaces of the cubic polynomials, Fractals, (6)(2)(1998), 181-189.

[24] S. Thianwan, Common xed points of new iterations for two asymptotically nonexpansive nonself mappings in a Banach space, $J$. Comput. Appl. Math. 224; (2009); 688-695.

[25] S. S. Dragomir and S. Fitzpatrick, The Hadamards inequality for s-convex functions in the second sense, Demonstr. Math. 32(4); (1999), 687-696.

[26] T. Lei, Similarity between the Mandelbrot sets and Julia sets, Commun. Math. Phys., (134)(3)(1990), $587-617$.

[27] U. S. Kirmaci,M. Klaricic Bakula,M.E. Ozdemir, and J. Pecaric, Hadamard-type inequalities for s-convex functions, Applied Mathematics and Computation, vol. 193, no. 1,(2007), 26-35.

[28] W. Nazeer, S. M. Kang, M. Tanveer and A. A. Shahid, Fixed point results in the generation of Julia and Mandelbrot sets, Journal of Inequalities and Applications, 2015 : 298; (2015), 1-16.

[29] W. Phuengrattana and S. Suantai, On the rate of convergence of Mann Ishikawa, Noor and SP-iterations for continuous functions on an arbitrary interval, Journal of Computational and Applied Mathematics, (235)(2011), 3006-3014.

[30] W. Takahashi, A convexity in metric space and nonexpansive mappings, I. Kodai Mathematical Seminar Reports, vol. 22, no. 2, (1970), 142-149.

[31] W. W. Breckner and G. Orban, Continuity Properties of Rationally s - Convex Mappings with Values in Ordered Topological Linear Space, Babes, - Bolyai University, Kolozsvar, Romania, 1978.

[32] X. Wang and C. Luo, Generalized Julia sets from a non-analytic complex map-ping, Appl. Math. Comput., (181)(2006), 113-122.

[33] X. Wang and P. Chang, Research on fractal structure of generalized M-J sets utilized Lyapunov exponents and periodic scanning techniques, Appl. Math. Comput., (175)(2006),1007-1025.

[34] X. Wang, L. Wei and Y. Xuejig, Research on Brownian movement based on generalized Mandelbrot Julia sets from a class complex mapping system, Mod. Phys. Lett. B, (21)(20)(2007), 1321-1341.

[35] X. Wang, X. Zhang, Y. Sun and L. Fanping, Dynamics of the generalized M set on escape-line diagram, Appl. Math. Comput., $(206)(2008) ; 474-484$.

[36] X. Wang, Y. He and Y. Sun, Accurate computation of periodic regions centers in the general M-set with integer index number, Discre. Dynam. Natur. Soc. (2010),12pages.

[37] Y. H. Geum and K. G. Hare, Groebner basis, resultants and the generalized Mandelbrot set, Chaos, Solitons \& Fractals, (42)(2)(2009), 1016-1023.

[38] Y. X. Tian, Convergence of an Ishikawa type iterative scheme for asymptotically quasi-nonexpansive mappings, Computer \& Mathematics with Applications, vol. 49; no. 11-12; (2005) 1905-1912.

\section{AUTHOR PROFILE}

Sudesh Kumari is a regular research scholar in the Department of Mathematics, Maharshi Dayanand University, Rohtak. Her interest areas are Fractal Theory and Nonlinear Analysis.

Mandeep Kumari is a regular research scholar in the Department of Mathematics, Maharshi Dayanand University, Rohtak. Her interest areas are Nonlinear Analysis and Manifolds.

Renu Chugh is working as a professor at Maharshi Dayanand University, Rohtak. Her interest areas are Approximation Theory, Nonlinear Functional Analysis, Fuzzy Mathematics, Theory of Frames, Fractals and Chaos. 